\title{
Parametric Adaptive Radar Detector with Enhanced Mismatched Signals Rejection Capabilities
}

\author{
Chengpeng Hao, ${ }^{1}$ Bin Liu, ${ }^{2}$ Shefeng Yan, ${ }^{1}$ and Long Cai ${ }^{1}$ \\ ${ }^{1}$ Institute of Acoustics, Chinese Academy of Sciences, Beijing 100190, China \\ ${ }^{2}$ Department of Electrical and Computer Engineering, Duke University, Durham, NC 27708, USA
}

Correspondence should be addressed to Chengpeng Hao, haochengp@sohu.com

Received 12 August 2010; Accepted 2 November 2010

Academic Editor: M. Greco

Copyright (C) 2010 Chengpeng Hao et al. This is an open access article distributed under the Creative Commons Attribution License, which permits unrestricted use, distribution, and reproduction in any medium, provided the original work is properly cited.

\begin{abstract}
We consider the problem of adaptive signal detection in the presence of Gaussian noise with unknown covariance matrix. We propose a parametric radar detector by introducing a design parameter to trade off the target sensitivity with sidelobes energy rejection. The resulting detector merges the statistics of Kelly's GLRT and of the Rao test and so covers Kelly's GLRT and the Rao test as special cases. Both invariance properties and constant false alarm rate (CFAR) behavior for this detector are studied. At the analysis stage, the performance of the new receiver is assessed and compared with several traditional adaptive detectors. The results highlight better rejection capabilities of this proposed detector for mismatched signals. Further, we develop two two-stage detectors, one of which consists of an adaptive matched filter (AMF) followed by the aforementioned detector, and the other is obtained by cascading a GLRT-based Subspace Detector (SD) and the proposed adaptive detector. We show that the former two-stage detector outperforms traditional two-stage detectors in terms of selectivity, and the latter yields more robustness.
\end{abstract}

\section{Introduction}

Adaptive detection of signals embedded in Gaussian or nonGaussian disturbance with unknown covariance matrix has been an active research field in the last few decades. Several generalized likelihood ratio test- (GLRT-) based methods are proposed, which utilize secondary (training) data, that is, data vectors sharing the same spectral properties, to form an estimate of the disturbance covariance. In particular, Kelly [1] derives a constant false alarm rate (CFAR) test for detecting target signals known up to a scaling factor; Robey et al. [2] develops a two-step GLRT design procedure, called adaptive matched filter (AMF). Based on the above methods, some improved approaches have been proposed, for example, the non-Gaussian version of Robey's adaptive strategy in [3-6] and the extended targets version of Kelly's adaptive detection strategy in [7]. In addition, considering the presence of mutual coupling and near-field effects, De Maio et al. [8] redevises Kelly's GLRT detector and the AMF.

Most of the above methods work well, provided that the exact knowledge of the signal array response vector is available; however, they may experience a performance degradation in practice when the actual steering vector is not aligned with the nominal one. A side lobe mismatched signal may appear subject to several causes, such as calibration and pointing errors, imperfect antenna shape, and wavefront distortions. To handle such mismatched signals, the Adaptive Beamformer Orthogonal Rejection Test (ABORT) [9] is proposed, which takes the rejection capabilities into account at the design stage, introducing a tradeoff between the detection performance for main lobe signals and rejection capabilities for side lobe ones. The directivity of this detector is in between that of the Kelly's GLRT and the Adaptive Coherence Estimator (ACE) $[10,11]$. A Whitened ABORT (W-ABORT) $[12,13]$ is proposed to address adaptive detection of distributed targets embedded in homogeneous disturbance via GLRT and the useful and fictitious signals orthogonal in the whitened space, which has an enhanced rejection capability for side lobe signals. Some alternative approaches are devised [14-17], which basically depend on constraining the actual signature to span a cone, whose axis coincides with its nominal value. Moreover, in [18], 
a detector based on the Rao test criterion is introduced and assessed. It is worth noting that the Rao test exhibits discrimination capabilities of mismatched signals better than those of the ABORT, although it does not consider a possible spatial signature mismatch at the design stage.

From another point of view, increased robustness to mismatch signals can be obtained by two-stage tunable receivers that are formed by cascading two detectors (usually with opposite behaviors), in which case, only data vectors exceeding both detection thresholds will be declared as the target bearings [19-23]. Remarkably, such solutions can adjust directivity by proper selection of the two thresholds to trade good rejection capabilities of side lobe signals for an acceptable detection loss for matched signals. An alternative approach to design tunable receivers relies on the parametric adaptive detectors, which allow us to trade off target sensitivity with side lobes energy rejection via tuning a design parameter [24, 25]. In particular, in [24], Kalson devises a parametric detector obtained by merging the statistics of Kelly's GLRT and of the AMF, whereas in [25], Bandiera et al. propose another parametric adaptive detector, which is obtained by mixing the statistic of Kelly's GLRT with that of the W-ABORT.

In this paper, we attempt to increase the rejection capabilities of tunable receivers and develop a novel adaptive parametric detector, which is obtained by merging the statistics of the Kelly's GLRT and of the Rao test. We show that the proposed detector is invariant under the group of transformations defined in [26]. As a consequence, it ensures the CFAR property with respect to the unknown covariance matrix of the noise. The performance assessment, conducted analytically for matched and mismatched signals, highlights that specified with a appropriate design parameter the new detector has better rejection capabilities for side lobe targets than existing decision schemes. However, if the value of the design parameter is bigger than or equals to unity, this new detector leads to worse detection performance than Kelly's receiver. To circumvent this drawback, a two-stage detector is proposed, which consists of the AMF followed by the proposed parametric adaptive detector and can be taken as an improved alternative of the two-stage detector in [18]. We also give another two-stage detector with enhanced robustness, which is obtained by cascading the GLRT-based Subspace Detector (SD) [27] and the proposed parametric adaptive receiver.

The paper is organized as follows. In the next section, we formulate the problem and then propose the adaptive parametric detector. In Section 3, we analyze the performance of the proposed receiver. We present two newly proposed two-stage tunable detectors, respectively, in Sections 4 and 5. Section 6 contains conclusions and avenues for further research. Finally, some analytical derivations are given in the Appendix.

\section{Problem Formulation and Design Issues}

We assume that data are collected from $N$ sensors and denote by $\mathbf{x} \in \mathbb{C}^{N \times 1}$ the complex vector of the samples where the presence of the useful signal is sought (primary data). As customary, we also suppose that a secondary data set $\mathbf{x}_{l}$, $l=1, \ldots, K$, is available $(K \geq N)$, that each of such snapshots does not contain any useful target echo and exhibits the same covariance matrix as the primary data (homogeneous environment).

The detection problem at hand can be formulated in terms of the following binary hypothesis test:

$$
\begin{aligned}
& H_{0}:\left\{\begin{array}{l}
\mathbf{x}=\mathbf{n}, \\
\mathbf{x}_{l}=\mathbf{n}_{l}, \quad l=1, \ldots, K,
\end{array}\right. \\
& H_{1}:\left\{\begin{array}{l}
\mathbf{x}=\alpha \mathbf{p}+\mathbf{n}, \\
\mathbf{x}_{l}=\mathbf{n}_{l}, \quad l=1, \ldots, K,
\end{array}\right.
\end{aligned}
$$

where

(i) $\mathbf{n}$ and $\mathbf{n}_{l} \in \mathbb{C}^{N \times 1}, l=1, \ldots, K$, are independent, complex, zero-mean Gaussian vectors with covariance matrix given by

$$
E\left[\mathbf{n n}^{\dagger}\right]=E\left[\mathbf{n}_{l} \mathbf{n}_{l}^{\dagger}\right]=\mathbf{M}, \quad l=1, \ldots, K,
$$

where $E[\cdot]$ denotes expectation and ${ }^{\dagger}$ conjugate transposition;

(ii) $\mathbf{p} \in \mathbb{C}^{N \times 1}$ is the unit-norm steering vector of main lobe target echo, which is possibly different from that of the nominal steering vector $\mathbf{p}_{0}$;

(iii) $\alpha \in \mathbb{C}$ is an unknown deterministic factor which accounts for both target reflectivity and channel effects.

The Rao test for the above problem [18] is given by

$$
\begin{aligned}
& t_{\text {rao }} \\
& =\frac{\left|\mathbf{x}^{\dagger} \mathbf{S}^{-1} \mathbf{p}_{0}\right|^{2}}{\left(1+\mathbf{x}^{\dagger} \mathbf{S}^{-1} \mathbf{x}\right) \mathbf{p}_{0}^{\dagger} \mathbf{S}^{-1} \mathbf{p}_{0}\left(1+\mathbf{x}^{\dagger} \mathbf{S}^{-1} \mathbf{x}-\left|\mathbf{x}^{\dagger} \mathbf{S}^{-1} \mathbf{p}_{0}\right|^{2} / \mathbf{p}_{0}^{\dagger} \mathbf{S}^{-1} \mathbf{p}_{0}\right)},
\end{aligned}
$$

where $\mathbf{S} \in \mathbb{C}^{N \times N}$ is $K$ times the sample covariance matrix of the secondary data, that is, $\mathbf{S}=\sum_{l=1}^{K} \mathbf{x}_{l} \mathbf{x}_{l}^{\dagger}$. It is straightforward to show that $t_{\text {rao }}$ can be recast as

$$
\begin{aligned}
t_{\text {rao }}= & \frac{t_{\text {glrt }}^{2}}{t_{\mathrm{amf}}\left(1-t_{\mathrm{glrt}}\right)} \\
= & \frac{t_{\mathrm{glrt}}}{t_{\mathrm{amf}}\left(1-t_{\mathrm{glrt}}\right)} t_{\mathrm{glrt}} \\
= & \left(1+\mathbf{x}^{\dagger} \mathbf{S}^{-1} \mathbf{x}-\frac{\left|\mathbf{x}^{\dagger} \mathbf{S}^{-1} \mathbf{p}_{0}\right|^{2}}{\mathbf{p}_{0}^{\dagger} \mathbf{S}^{-1} \mathbf{p}_{0}}\right)^{-1} \\
& \times \frac{\left|\mathbf{x}^{\dagger} \mathbf{S}^{-1} \mathbf{p}_{0}\right|^{2}}{\left(1+\mathbf{x}^{\dagger} \mathbf{S}^{-1} \mathbf{x}\right)\left(\mathbf{p}_{0}^{\dagger} \mathbf{S}^{-1} \mathbf{p}_{0}\right)}
\end{aligned}
$$


where

$$
t_{\mathrm{amf}}=\frac{\left|\mathbf{x}^{\dagger} \mathbf{S}^{-1} \mathbf{p}_{0}\right|^{2}}{\mathbf{p}_{0}^{\dagger} \mathbf{S}^{-1} \mathbf{p}_{0}}
$$

is the AMF decision statistic, and

$$
t_{\mathrm{glrt}}=\frac{\left|\mathbf{x}^{\dagger} \mathbf{S}^{-1} \mathbf{p}_{0}\right|^{2}}{\left(1+\mathbf{x}^{\dagger} \mathbf{S}^{-1} \mathbf{x}\right)\left(\mathbf{p}_{0}^{\dagger} \mathbf{S}^{-1} \mathbf{p}_{0}\right)}
$$

is the decision statistic of Kelly's GLRT.

Comparing $t_{\text {rao }}$ with $t_{\text {glrt }}$, we propose a new detector, termed KRAO in the following. Its decision statistic is

$$
\begin{aligned}
t_{\mathrm{krao}}= & \left(1+\mathbf{x}^{\dagger} \mathbf{S}^{-1} \mathbf{x}-\frac{\left|\mathbf{x}^{\dagger} \mathbf{S}^{-1} \mathbf{p}_{0}\right|^{2}}{\mathbf{p}_{0}^{\dagger} \mathbf{S}^{-1} \mathbf{p}_{0}}\right)^{-(2 \rho-1)} \\
& \times \frac{\left|\mathbf{x}^{\dagger} \mathbf{S}^{-1} \mathbf{p}_{0}\right|^{2}}{\left(1+\mathbf{x}^{\dagger} \mathbf{S}^{-1} \mathbf{x}\right)\left(\mathbf{p}_{0}^{\dagger} \mathbf{S}^{-1} \mathbf{p}_{0}\right)}
\end{aligned}
$$

or, equivalently

$$
t_{\text {krao }}=\left[\frac{t_{\mathrm{glrt}}}{t_{\mathrm{amf}}\left(1-t_{\mathrm{glrt}}\right)}\right]^{(2 \rho-1)} t_{\mathrm{glrt}}
$$

where $\rho$ is the design parameter.

It is clear that our detector covers Kelly's GLRT and the Rao test as special cases, respectively, when $\rho=0.5$ and $\rho=1$. Moreover, since $t_{\text {krao }}$ can be expressed in terms of the maximal invariant statistic $\left(t_{\mathrm{amf}}, t_{\mathrm{glrt}}\right)$, it is invariant with respect to the transformations defined in [26]. As a consequence, it ensures the CFAR property with respect to the unknown covariance matrix of the noise.

\section{Performance Assessment}

In this section, we derive an analytic expression of $P_{f a}$ and $P_{d}$ and then present illustrative examples for KRAO. Specifically, in derivation of $P_{d}$, we consider a general case, in which the signal in the primary data vector is not commensurate with the nominal steering vector, that is we consider detection performance for mismatched signal. To this end, we first introduce the random variable

$$
\beta=\left(1+\mathbf{x}^{\dagger} \mathbf{S}^{-1} \mathbf{x}-\frac{\left|\mathbf{x}^{\dagger} \mathbf{S}^{-1} \mathbf{p}_{0}\right|^{2}}{\mathbf{p}_{0}^{\dagger} \mathbf{S}^{-1} \mathbf{p}_{0}}\right)^{-1}
$$

and then consider the equivalent form of Kelly's statistic $\hat{t}_{\mathrm{glrt}}=t_{\mathrm{glrt}} /\left(1-t_{\mathrm{glrt}}\right)$. Thus, $t_{\mathrm{krao}}$ can be expressed to be

$$
t_{\text {krao }}=\beta^{2 \rho-1} \frac{\hat{t}_{\mathrm{glrt}}}{1+\hat{t}_{\mathrm{glrt}}} .
$$

3.1. $P_{f a}$ of the KRAO. Under $H_{0}$ hypothesis, the following statements hold [21]:

(i) given $\beta, \hat{t}_{\text {glrt }}$ is ruled by the complex central $\mathrm{F}$ distribution with $1, K-N+1$ degrees of freedom, namely, $\hat{t}_{\mathrm{glrt}} \sim \mathcal{C} \mathcal{F}_{1, K-N+1}$; (ii) $\beta$ is a complex central beta distribution random variable (rv) with $K-N+2, N-1$ degrees of freedom, namely, $\beta \sim \mathcal{C} \beta_{K-N+2, N-1}$.

Therefore, the KRAO associated $P_{f a}$ satisfies

$$
\begin{aligned}
P_{f a}(\rho, \eta) & =P\left(\beta^{2 \rho-1} \frac{\hat{t}_{\mathrm{glrt}}}{1+\hat{t}_{\mathrm{glrt}}}>\eta ; H_{0}\right) \\
& =P\left(\hat{t}_{\mathrm{glrt}}>\frac{\eta}{\beta^{2 \rho-1}-\eta} ; H_{0}\right) \\
& =\int_{0}^{1}\left[1-F_{0}\left(\frac{\eta}{\varepsilon^{2 \rho-1}-\eta}\right)\right] f_{\beta}(\varepsilon) d \varepsilon
\end{aligned}
$$

where $\eta$ is the threshold set beforehand, whose value depends on the value of $P_{f a}, f_{\beta}(\cdot)$ is the probability density function (pdf) of the $\operatorname{rv} \beta \sim \mathcal{C} \beta_{K-N+2, N-1}$, and $F_{0}(\cdot)$ is the cumulative

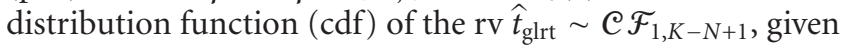
$\beta$. Then it follows

$$
\begin{aligned}
& P\left(\frac{\hat{t}_{\mathrm{glrt}}}{1+\hat{t}_{\mathrm{glrt}}}>\frac{\eta}{\beta^{2 \rho-1}} ; H_{0}\right) \\
& \quad= \begin{cases}0, & \beta^{2 \rho-1} \leq \eta \\
P\left(\hat{t}_{\mathrm{glrt}}>\frac{\eta}{\beta^{2 \rho-1}-\eta} ; H_{0}\right), & \beta^{2 \rho-1}>\eta\end{cases}
\end{aligned}
$$

Substituting (12) into (11) followed by some algebra, it yields

(i) $\rho \geq 0.5$ and $\eta \geq 1$

$$
P_{f a}(\rho, \eta)=0
$$

(ii) $\rho>0.5$ and $0 \leq \eta<1$

$$
P_{f a}(\rho, \eta)=\int_{\eta^{1 /(2 \rho-1)}}^{1}\left[1-F_{0}\left(\frac{\eta}{\varepsilon^{2 \rho-1}-\eta}\right)\right] f_{\beta}(\varepsilon) d \varepsilon,
$$

(iii) $0 \leq \rho<0.5$ and $\eta \geq 1$

$$
P_{f a}(\rho, \eta)=\int_{0}^{\eta^{1 /(2 \rho-1)}}\left[1-F_{0}\left(\frac{\eta}{\varepsilon^{2 \rho-1}-\eta}\right)\right] f_{\beta}(\varepsilon) d \varepsilon,
$$

(iv) $0 \leq \rho \leq 0.5$ and $0 \leq \eta<1$

$$
P_{f a}(\rho, \eta)=\int_{0}^{1}\left[1-F_{0}\left(\frac{\eta}{\varepsilon^{2 \rho-1}-\eta}\right)\right] f_{\beta}(\varepsilon) d \varepsilon
$$

For the reader ease, Figure 1 shows the contour plots for the KRAO corresponding to different values of $P_{f a}$, as functions of the threshold pairs $(\rho, \eta), N=8$, and $K=$ 24 . All curves have been obtained by means of numerical integration techniques. 


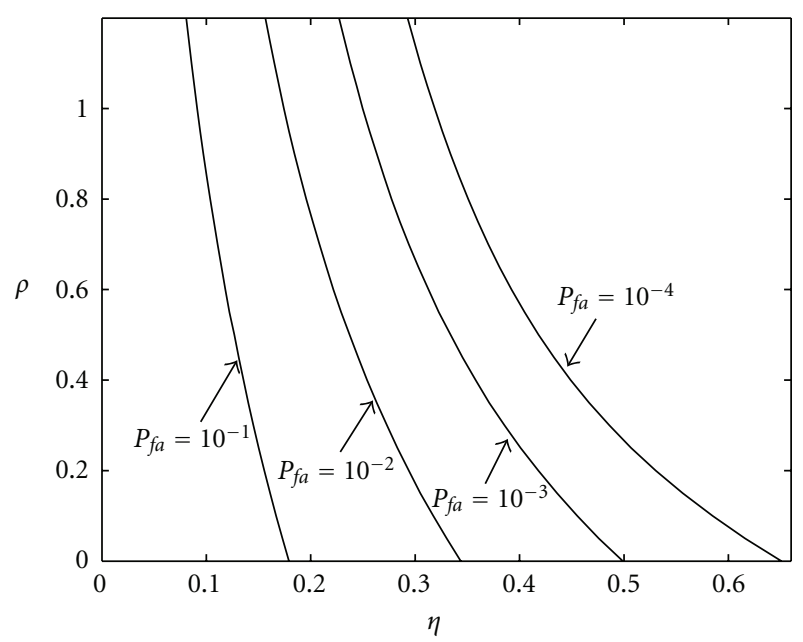

Figure 1: Contours of constant $P_{f a}$ for the KRAO versus $\eta$ and $\rho$ with $N=8, K=24$.

3.2. $P_{d}$ of the KRAO. Now we consider hypothesis $H_{1}$. Denote $\phi$ the angle between $\mathbf{p}$ and $\mathbf{p}_{0}$ in the whiteneddimensional data space, that is,

$$
\cos ^{2} \phi=\frac{\left|\mathbf{p}^{\dagger} \mathbf{M}^{-1} \mathbf{p}_{0}\right|^{2}}{\left(\mathbf{p}^{\dagger} \mathbf{M}^{-1} \mathbf{p}\right)\left(\mathbf{p}_{0}^{\dagger} \mathbf{M}^{-1} \mathbf{p}_{0}\right)}
$$

The term $\cos ^{2} \phi$ is a measure of the mismatch between $\mathbf{p}$ and $\mathbf{p}_{0}$. Its value is one for the matched case where $\mathbf{p}=\mathbf{p}_{\mathbf{0}}$, and less than one otherwise. A small value of $\cos ^{2} \phi$ implies a large mismatch between the steering vector and signal. In this case, due to the useful signal components, distributions of $\hat{t}_{\mathrm{glrt}}$ and $\beta$ are given in [23]:

(i) given $\beta, \hat{t}_{\text {glrt }}$ is ruled by the complex noncentral Fdistribution with $1, K-N+1$ degrees of freedom and noncentrality parameter

$$
\delta_{\phi}^{2}=\beta \mathrm{SNR} \cos ^{2} \phi
$$

namely, $\hat{t}_{\text {glrt }} \sim \mathcal{C} \mathcal{F}_{1, K-N+1}\left(\delta_{\phi}\right)$, where SNR $=$ $|\alpha|^{2} \mathbf{p}^{\dagger} \mathbf{M}^{-1} \mathbf{p}$ is the total available signal-to-noise ratio;

(ii) $\beta$ is a complex noncentral beita distribution rv with $K-N+2, N-1$ degrees of freedom and noncentrality parameter

$$
\delta_{\beta}^{2}=\operatorname{SNR} \sin ^{2} \phi,
$$

namely, $\beta \sim \mathcal{C} \beta_{K-N+2, N-1}\left(\delta_{\beta}\right)$.

Then $P_{d}$ is given by

$$
\begin{aligned}
P_{d}(\phi) & =P\left(\beta^{2 \rho-1} \frac{\hat{t}_{\mathrm{glrt}}}{1+\hat{t}_{\mathrm{glrt}}}>\eta ; H_{1}\right) \\
& =\int_{0}^{1}\left[1-F_{1}\left(\frac{\eta}{\varepsilon^{2 \rho-1}-\eta}\right)\right] f_{\beta}(\varepsilon) d \varepsilon,
\end{aligned}
$$

where $f_{\beta}(\cdot)$ is the pdf of the $\mathrm{rv} \beta \sim \mathcal{C} \beta_{K-N+2, N-1}\left(\delta_{\beta}\right)$, and then, given $\beta, F_{1}(\cdot)$ is the cdf of the $\operatorname{rv} \hat{t}_{\text {glrt }} \sim \mathcal{C} \mathcal{F}_{1, K-N+1}\left(\delta_{\phi}\right)$. Similarly as before (in Section 3.1), we have

(i) $\rho \geq 0.5$ and $\eta \geq 1$

$$
P_{d}(\phi)=0
$$

(ii) $\rho>0.5$ and $0 \leq \eta<1$

$$
P_{d}(\phi)=\int_{\eta^{1 /(2 \rho-1)}}^{1}\left[1-F_{1}\left(\frac{\eta}{\varepsilon^{2 \rho-1}-\eta}\right)\right] f_{\beta}(\varepsilon) d \varepsilon,
$$

(iii) $0 \leq \rho<0.5$ and $\eta \geq 1$

$$
P_{d}(\phi)=\int_{0}^{\eta^{1 /(2 \rho-1)}}\left[1-F_{1}\left(\frac{\eta}{\varepsilon^{2 \rho-1}-\eta}\right)\right] f_{\beta}(\varepsilon) d \varepsilon,
$$

(iv) $0 \leq \rho \leq 0.5$ and $0 \leq \eta<1$

$$
P_{d}(\phi)=\int_{0}^{1}\left[1-F_{1}\left(\frac{\eta}{\varepsilon^{2 \rho-1}-\eta}\right)\right] f_{\beta}(\varepsilon) d \varepsilon .
$$

In the case of a perfect match, $\delta_{\beta}$ is equal to zero. As a consequence, $\beta$ is distributed as a complex central beta distribution random variable with $K-N+2, N-1$ degrees of freedom, and $\hat{t}_{\text {glrt }}$ is ruled by the complex noncentral F-distribution with $1, K-N+1$ degrees of freedom and noncentrality parameter

$$
\delta_{0}^{2}=\beta \mathrm{SNR}
$$

3.3. Performance Analysis. In this subsection, we present numerical examples to illustrate the performance of the KRAO. The curves are obtained by numerical integration and the probability of false alarm is set to $10^{-4}$.

One can see the influence of the design parameter $\rho$ in Figures 2 and 3, where the $P_{d}$ of the KRAO is plotted versus the SNR, considering both the case of a perfect match between the actual steering vector and the nominal one, namely, $\cos ^{2} \phi=1$, and the case where there is a misalignment between the two aforementioned vectors, more precisely $\cos ^{2} \phi=0.7$. Specifically, Figures 2 and 3 correspond to $\rho \geq 0.5$ and $\rho \in[0,0.5]$, respectively. From Figure 2, we see that the curves associated with the KRAO are in between that of Kelly's GLRT and that of the Rao test when $\rho \in(0.5,1.0)$, and that the KRAO outperforms the Rao test in terms of selectivity for $\rho>1$. However, it is also shown that the amount of detection loss for matched signals and sensitivity to mismatched signals depend upon the design parameter $\rho$. More specifically, a larger value of $\rho$ leads to better rejection capabilities of the side lobe signals and the larger detection loss for matched signals. On the other hand, Figure 3 shows that, when $\rho \in[0,0.5)$, a smaller value of $\rho$ renders the performance less sensitive to mismatched signals. In another word, robustness to mismatched signals can be increased by setting $\rho \in[0,0.5)$. In summary, different values of $\rho$ represent different compromises between the detection 


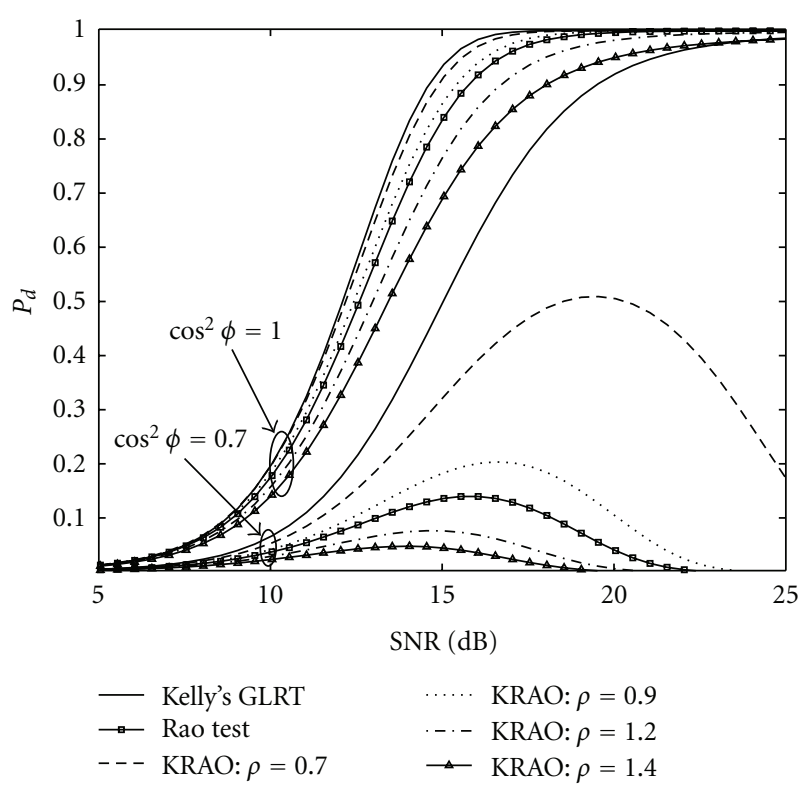

Figure 2: $P_{d}$ versus SNR for the KRAO, $N=8, K=24$, and $\rho \geq 0.5$.

and the rejection performance. So the appropriate value of $\rho$ is selected based on the system needs.

In Figures 4 and 5, we compare the KRAO to the ACE, the ABORT, and Bandiera's detector (KWA) [25] for $N=16$, $K=32$, and under the constraint that the loss with respect to Kelly's GLRT is practically the same for the perfectly matched case. For sake of completeness, we review these CFAR detectors in the following:

$$
\begin{aligned}
t_{\mathrm{ace}} & =\frac{\left|\mathbf{x}^{\dagger} \mathbf{S}^{-1} \mathbf{p}_{0}\right|^{2}}{\left(\mathbf{p}_{0}^{\dagger} \mathbf{S}^{-1} \mathbf{p}_{0}\right)\left(\mathbf{x}^{\dagger} \mathbf{S}^{-1} \mathbf{x}\right)}, \\
t_{\mathrm{abort}} & =\frac{1+\left|\mathbf{x}^{\dagger} \mathbf{S}^{-1} \mathbf{P}_{0}\right|^{2} / \mathbf{p}_{0}^{\dagger} \mathbf{S}^{-1} \mathbf{P}_{0}}{2+\mathbf{x}^{\dagger} \mathbf{S}^{-1} \mathbf{x}}, \\
t_{\mathrm{kwa}} & =\frac{1+\mathbf{x}^{\dagger} \mathbf{S}^{-1} \mathbf{x}}{\left[1+\mathbf{x}^{\dagger} \mathbf{S}^{-1} \mathbf{x}-\left|\mathbf{x}^{\dagger} \mathbf{S}^{-1} \mathbf{p}_{0}\right|^{2} /\left(\mathbf{p}_{0}^{\dagger} \mathbf{S}^{-1} \mathbf{p}_{0}\right)\right]^{2 \gamma}},
\end{aligned}
$$

where $\gamma$ is the design parameter of the KWA. From Figures 4 and 5 , it is clear that the KRAO is superior to the KWA in rejecting side lobe signals with $\rho=\gamma+0.1$ It is also clear that, with a proper choice of $\rho$, the KRAO outperforms the ACE and the ABORT in terms of selectivity. Other simulation results not reported here, in order not to burden too much the analysis, have shown that the above results are still valid for $N=8$ and $K=24$.

\section{Two-Stage Detector Based on the KRAO}

In this section, we propose a two-stage algorithm, aiming at compensating the matched detection performance loss for the KRAO with $\rho \geq 1$. Briefly, this is obtained by cascading the AMF and the KRAO $(\rho \geq 1)$. We term this two-stage detector KRAO Adaptive Side lobe Blanker (KRAO-ASB). This detector generalizes the two-stage Rao test (AMF-RAO)

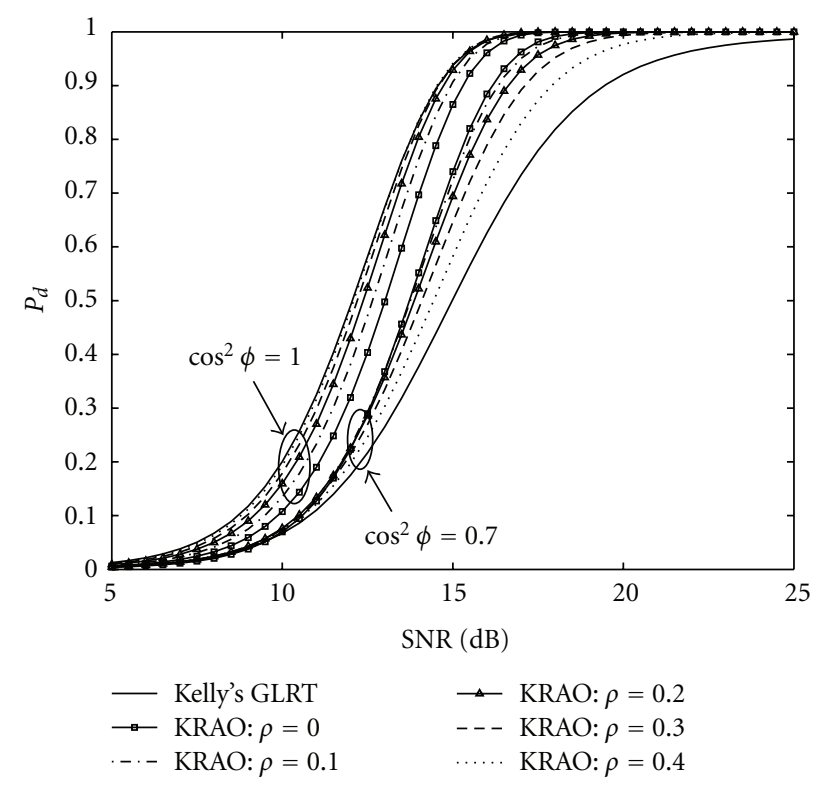

Figure 3: $P_{d}$ versus SNR for the KRAO, $N=8, K=24$, and $\rho \in$ $[0,0.5]$.

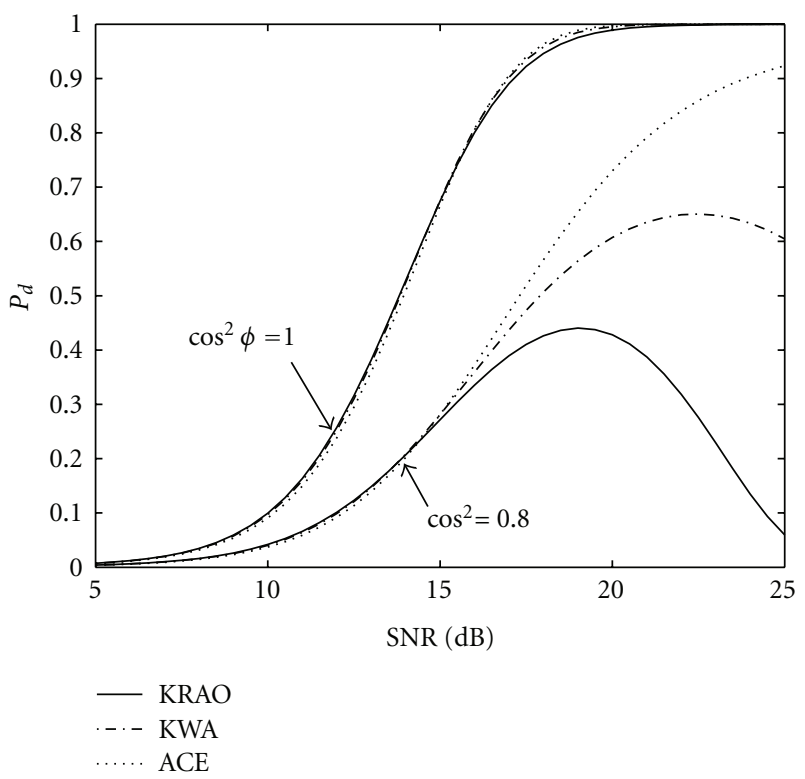

FIgure 4: $P_{d}$ versus SNR for the KRAO with $\rho=0.9$, the KWA with $\gamma=0.8$, and the ACE, $N=16, K=32$.

[18] for $\rho=1$. We now summarize the implementation of the proposed detector as below:

$$
\begin{array}{cc}
t_{\mathrm{amf}} \gtrless \eta_{a} \stackrel{>\eta_{a}}{\longrightarrow} & t_{\mathrm{krao}} \gtrless \eta_{k} \stackrel{>\eta_{k}}{\longrightarrow} H_{1} \\
\downarrow & \downarrow \leq \eta_{a} \\
H_{0} & H_{0},
\end{array}
$$

where $\eta_{a}$ and $\eta_{k}$ form the threshold pair, which are set in such a way that the desired $P_{f a}$ is available. Observe that the KRAO-ASB is invariant to the group of transformations given in [26], due to the fact that $t_{\mathrm{krao}}$ can be expressed 
in terms of the maximal invariant statistic $\left(t_{\mathrm{amf}}, t_{\mathrm{glrt}}\right)$. It is thus not surprising that the KRAO-ASB ensures the CFAR property with respect to the disturbance covariance matrix M. In what follows, we derive the closed-form expressions for $P_{f a}$ and $P_{d}$ of KRAO-ASB. Given a stochastic representation for $t_{\mathrm{amf}}[20]$ :

$$
t_{\mathrm{amf}}=\frac{\hat{t}_{\mathrm{glrt}}}{\beta}
$$

the $P_{f a}$ follows to be

$$
\begin{aligned}
P_{f a}\left(\eta_{a}, \eta_{k}, \rho\right) & =P\left[t_{\mathrm{amf}}>\eta_{a}, t_{\mathrm{krao}}>\eta_{k} ; H_{0}\right] \\
& =P\left[\frac{\hat{t}_{\mathrm{glrt}}}{\beta}>\eta_{a}, \beta^{2 \rho-1} \frac{\hat{t}_{\mathrm{glrt}}}{1+\hat{t}_{\mathrm{glrt}}}>\eta_{k} ; H_{0}\right] \\
& =P\left[\hat{t}_{\mathrm{glrt}}>\max \left(\beta \eta_{a}, \frac{\eta_{k}}{\beta^{2 \rho-1}-\eta_{k}}\right) ; H_{0}\right] .
\end{aligned}
$$

Note that

$$
P_{f a}\left(\eta_{a}, \eta_{k}, \rho\right)= \begin{cases}0, & \beta \leq \eta_{k}^{1 /(2 \rho-1)}, \\ \max \left(\beta \eta_{a}, \frac{\eta_{k}}{\beta^{2 \rho-1}-\eta_{k}}\right), & \beta>\eta_{k}^{1 /(2 \rho-1)} .\end{cases}
$$

Consequently,

$$
\begin{aligned}
& P_{f a}\left(\eta_{a}, \eta_{k}, \rho\right) \\
& =\int_{\eta_{k}^{1 /(2 \rho-1)}}^{1} P\left[\hat{t}_{\mathrm{glrt}}>\max \left(x \eta_{a}, \frac{\eta_{k}}{x^{2 \rho-1}-\eta_{k}}\right) \mid \beta=x ; H_{0}\right] \\
& \quad \times f_{\beta}(x) d x \\
& =\int_{\eta_{k}^{1 /(2 \rho-1)}}^{1}\left[1-F_{0}\left(\max \left(x \eta_{a}, \frac{\eta_{k}}{x^{2 \rho-1}-\eta_{k}}\right)\right)\right] f_{\beta}(x) d x
\end{aligned}
$$

where $f_{\beta}(\cdot)$ is pdf of the $\operatorname{rv} \beta \sim \mathcal{C} \beta_{K-N+2, N-1}$, and $F_{0}(\cdot)$ is the cdf of the rv $\hat{t}_{\text {glrt }} \sim \mathcal{C} \mathcal{F}_{1, K-N+1}$, given $\beta$. Then, we consider the standard algebra

$$
\max \left(x \eta_{a}, \frac{\eta_{k}}{x^{2 \rho-1}-\eta_{k}}\right)= \begin{cases}x \eta_{a}, & x>\sigma \\ \frac{\eta_{k}}{x^{2 \rho-1}-\eta_{k}}, & x \leq \sigma,\end{cases}
$$

where $\sigma$ is the positive root to the equation

$$
\eta_{a} x^{2 \rho-1}-\eta_{a} \eta_{k} x-\eta_{k}=0
$$

and can be obtained via Newton's method. Substituting (32) into (31) and performing some algebra, it yields that

(i) if $\eta_{a} \leq \eta_{k} /\left(1-\eta_{k}\right)$, then $\sigma \geq 1$

$$
P_{f a}\left(\eta_{a}, \eta_{k}, \rho\right)=\int_{\eta_{k}^{1 /(2 \rho-1)}}^{1}\left[1-F_{0}\left(\frac{\eta_{k}}{x^{2 \rho-1}-\eta_{k}}\right)\right] f_{\beta}(x) d x
$$

namely, the two-stage detector achieves the same performance as that of the KRAO test;

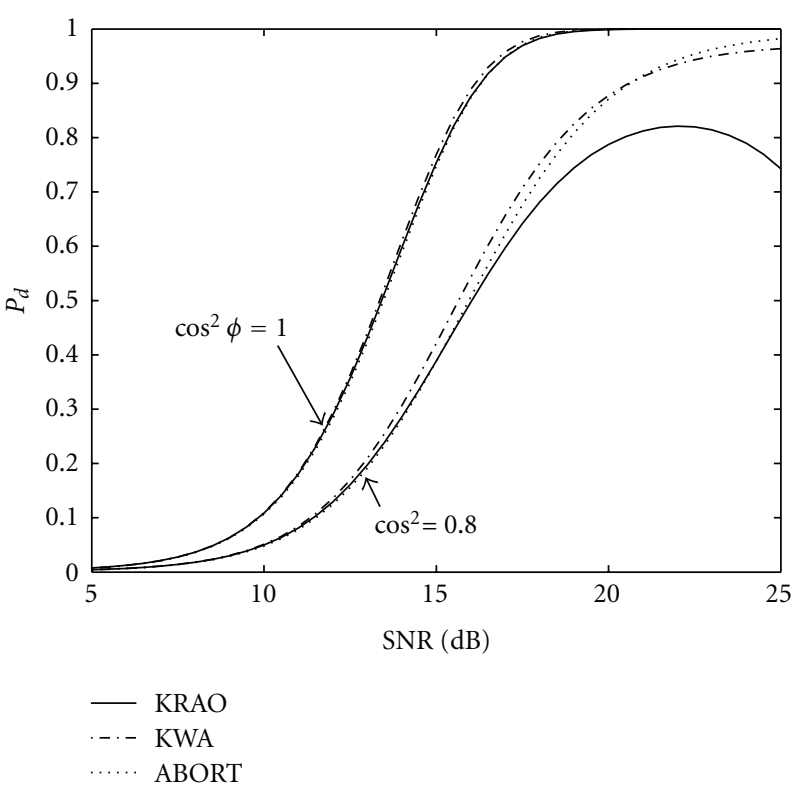

FIgURe 5: $P_{d}$ versus SNR for the KRAO with $\rho=0.7$, the KWA with $\gamma=0.6$, and the ABORT, $N=16, K=32$.

(ii) if $\eta_{a}>\eta_{k} /\left(1-\eta_{k}\right)$, then $\sigma<1$

$$
\begin{aligned}
P_{f a}\left(\eta_{a}, \eta_{k}, \rho\right)= & \int_{\eta_{k}^{1 /(2 \rho-1)}}^{\sigma}\left[1-F_{0}\left(\frac{\eta_{k}}{x^{2 \rho-1}-\eta_{k}}\right)\right] f_{\beta}(x) d x \\
& +\int_{\sigma}^{1}\left(1-F_{0}\left(x \eta_{a}\right)\right) f_{\beta}(x) d x .
\end{aligned}
$$

It is worth noting that there exist an infinite set of infinite triplets $\left(\eta_{a}, \eta_{k}, \rho\right)$ that result in the same $P_{f a}$. Figure 6 shows the contour plots corresponding to different values of $P_{f a}$, as functions of $\left(\eta_{a}, \eta_{k}\right)$ for $N=8, K=24$, and $\rho=1.2$. It is shown that this detector provides a compromise between the detection and the rejection performance and degenerates to the AMF as $\eta_{k}=0$, and the KRAO when $\eta_{a}=0$. So the appropriate operating point can be selected based on the system requirements.

For $H_{1}$ hypothesis, the derivation process is similar. In detail, if $\eta_{a} \leq \eta_{k} /\left(1-\eta_{k}\right), P_{d}$ is the same as for the KRAO test; otherwise, it can be evaluated by

$$
\begin{aligned}
P_{d}(\phi)= & \int_{\eta_{k}^{1 /(2 \rho-1)}}^{\sigma}\left[1-F_{1}\left(\frac{\eta_{k}}{x^{2 \rho-1}-\eta_{k}}\right)\right] f_{\beta}(x) d x \\
& +\int_{\sigma}^{1}\left[1-F_{1}\left(x \eta_{a}\right)\right] f_{\beta}(x) d x
\end{aligned}
$$

where $f_{\beta}(\cdot)$ is the pdf of the $\operatorname{rv} \beta \sim \mathcal{C} \beta_{K-N+2, N-1}\left(\delta_{\beta}\right)$, and $F_{1}(\cdot)$ is the cdf of the $\mathrm{rv} \hat{t}_{\text {glrt }} \sim \mathcal{C} \mathcal{F}_{1, K-N+1}\left(\delta_{\phi}\right)$, given $\beta$.

The matched detection performances of the KRAO-ASB, the KRAO, and the AMF are analyzed in Figure 7, with $N=$ $8, K=24, \rho=1.2$, and $P_{f a}=10^{-4}$. For KRAO-ASB, we show the curve corresponding to the threshold setting that returns the minimum loss with respect to the Kelly's GLRT. 


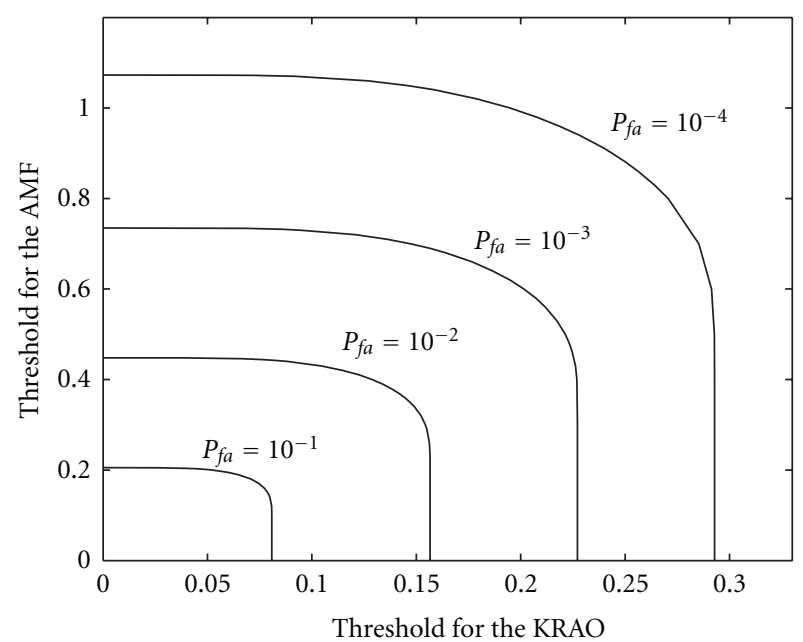

Figure 6: Contours of constant $P_{f a}$ for the KRAO-ASB with $N=8$, $K=24$, and $\rho=1.2$.

The curves highlight that for small-medium SNR values, the KRAO-ASB yields better detection performance than that obtained by performing either the AMF or the KRAO operating alone. We argue that this behavior results from the capability of the KRAO-ASB algorithm in combining information from both single detectors. Similar results for existing two-stage detectors refer to [18-21].

In Figures 8 and 9 , we compare the KRAO-ASB (equipped with $\rho=1.2$ ) to the two-stage detector based on the KWA (KWAS-ASB) [25] (affiliated with $\gamma=1.1$ ) and the AMF-RAO. The threshold pairs correspond to the most selective case and entail a loss for matched signals of about $1 \mathrm{~dB}$ with respect to the Kelly's GLRT at $P_{d}=0.9$ and $P_{f a}=10^{-4}$. Figure 8 refers to $N=8$ and $K=24$, and Figure 9 assumes $N=16$ and $K=32$. As it can be seen, the KRAO-ASB exhibits better rejection capabilities of mismatched signals than the KWAS-ASB and the AMF-RAO for the considered system parameters.

\section{Improved Two-Stage Detector Based on the KRAO}

In order to increase the robustness to mismatched signals of the KRAO-ASB, we propose another two-stage detector. This detector is the same as KRAO-ASB, except that the AMF is replaced by a SD. The resulting statistic is

$$
t_{s d}=\frac{\mathbf{x}^{\dagger} \mathbf{S}^{-1} \mathbf{H}\left(\mathbf{H}^{\dagger} \mathbf{S}^{-1} \mathbf{H}\right)^{-1} \mathbf{H}^{\dagger} \mathbf{S}^{-1} \mathbf{x}}{1+\mathbf{x}^{\dagger} \mathbf{S}^{-1} \mathbf{x}},
$$

where $\mathbf{H}=\left[\mathbf{v} \cdots \mathbf{v}_{r-1}\right] \in \mathbb{C}^{N \times r}$ is a full-column-rank matrix $(r \geq 1)$. The choice of $\mathbf{H}=[\mathbf{s}(0), \mathbf{s}(\pi / 360)]$ makes this detector robust in a homogeneous environment [21]. The vector $\mathbf{s}(\theta)$ is defined as follows:

$$
\mathbf{s}(\theta)=\frac{1}{\sqrt{N}}\left[1, e^{j(2 \pi d / \lambda) \sin \theta}, \ldots, e^{j(N-1)(2 \pi d / \lambda) \sin \theta}\right]^{T},
$$

where $\lambda$ is the radar operating wavelength, $d$ is the interelement spacing, and $T$ denotes transposition.
This detector, which we term Subspace-based and KRAO Adaptive Side lobe Blanker (SKRAO-ASB), can be pictorially described as follows:

$$
\begin{array}{cc}
t_{s d} \gtrless \eta_{s} \stackrel{>\eta_{s}}{\longrightarrow} & t_{\text {krao }} \gtrless \eta_{k} \stackrel{>\eta_{k}}{\longrightarrow} H_{1} \\
\downarrow \leq \eta_{s} & \downarrow \leq \eta_{k} \\
H_{0} & H_{0},
\end{array}
$$

where $\eta_{s}$ and $\eta_{k}$ form the threshold pair which should be set beforehand to guarantee that the overall desired $P_{f a}$ is available. We then derive closed-form expressions for $P_{f a}$ and $P_{d}$ of the KRAOS-ASB. First, we replace $t_{s d}$ with the equivalent decision statistic $\hat{t}_{s d}=1 /\left(1-t_{s d}\right)$. It is shown that the following identities hold for $\hat{t}_{s d}$ and $t_{k \text { rao }}$ (see derivation in Appendix):

$$
\begin{aligned}
\hat{t}_{s d} & =(1+c) \hat{t}_{\mathrm{glrt}}, \\
t_{\mathrm{krao}} & =\left(\frac{1}{1+b+c+b c}\right)^{2 \rho-1} \frac{\hat{t}_{\mathrm{glrt}}}{1+\hat{t}_{\mathrm{glrt}}}
\end{aligned}
$$

Then, under $H_{0}$ hypothesis [23]:

(i) given $b$ and $c, \hat{t}_{\text {glrt }}$ is ruled by the complex central Fdistribution with $1, K-N+1$ degrees of freedom, namely, $\hat{t}_{\text {glrt }} \sim \mathcal{C \mathcal { F } _ { 1 , K - N + 1 }}$

(ii) $b$ is a complex central F-distribution random variable (rv) with $N-r, K-N+r+1$ degrees of freedom, namely, $b \sim \mathcal{C} \mathcal{F}_{N-r, K-N+r+1}$;

(iii) $c$ obeys the complex central F-distribution with $r-$ $1, K-N+2$ degrees of freedom, namely, $c \sim$ $\mathcal{C} \mathcal{F}_{r-1, K-N+2}$;

(iv) $b$ and $c$ are statistically independent rv's.

Therefore, the $P_{f a}$ of the SKRAO-ASB can be expressed as

$$
\begin{aligned}
& P_{f a}\left(\eta_{s}, \eta_{r}, \rho\right)=P\left(\hat{t}_{s d}>\hat{\eta}_{s}, t_{k \mathrm{rao}}>\eta_{k} ; H_{0}\right) \\
&=\iint_{0}^{\infty}[ 1-F_{0}\left(\operatorname { m a x } \left(\frac{\hat{\eta}_{s}}{1+k}-1,\right.\right. \\
&\left.\left.\left.\frac{\eta_{k}}{(1+\varepsilon+k+\varepsilon k)^{1-2 \rho}-\eta_{k}}\right)\right)\right] \\
& \times f_{b}(\varepsilon) f_{c}(k) d \varepsilon \mathrm{d} k,
\end{aligned}
$$

where $\hat{\eta}_{s}=1 /\left(1-\eta_{s}\right), f_{b}(\cdot)$ is the pdf of the $\mathrm{rv} b \sim$

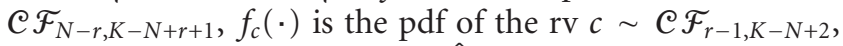
and $F_{0}(\cdot)$ is the cdf of the rv $\hat{t}_{\text {glrt }} \sim \mathcal{C} \mathcal{F}_{1, K-N+1}$, given $b$ and $c$. As can be seen from (41), the $P_{f a}$ of the SKRAOASB depends on the threshold pairs $\left(\hat{\eta}_{s}, \eta_{\mathrm{k}}\right)$ and the design parameter $\rho$, as a consequence of which, the SKRAO-ASB possesses the constant false alarm rate (CFAR) property with respect to the disturbance covariance matrix $\mathbf{M}$.

For hypothesis $H_{1}$, we assume that the first column of $\mathbf{H}$ is $\mathbf{p}_{0}$, then perform $Q R$ factorization to $\mathbf{M}^{-1 / 2} \mathbf{H}$ :

$$
\mathbf{M}^{-1 / 2} \mathbf{H}=\mathbf{H}_{0} \mathbf{R}_{H}
$$




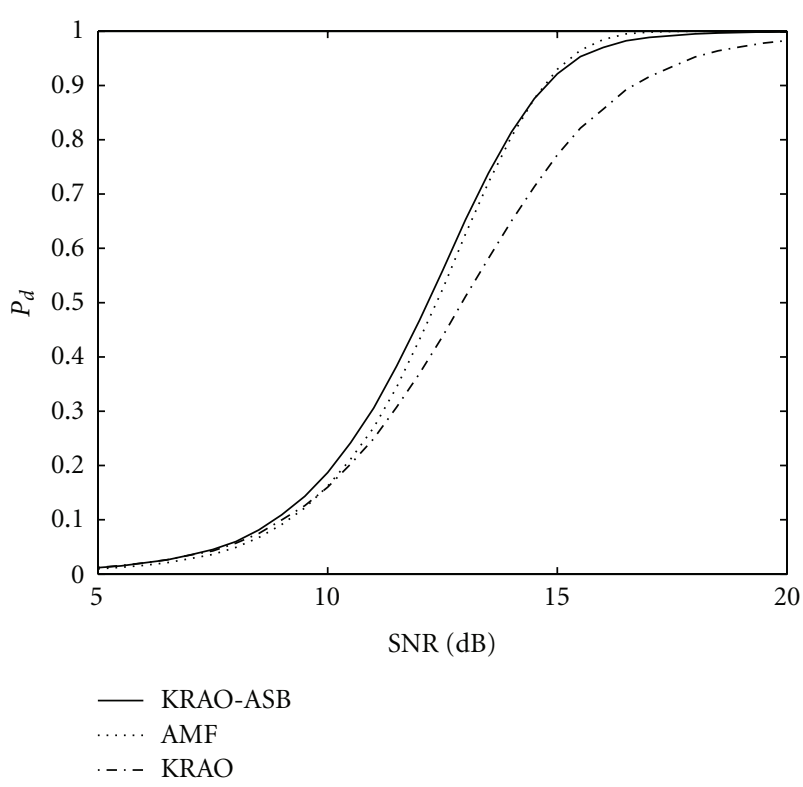

Figure 7: Matched $P_{d}$ versus SNR for the KRAO-ASB, the KRAO, and the AMF with $N=8, K=24$, and $\rho=1.2$.

with $\mathbf{H}_{0} \in \mathbb{C}^{N \times r}$ being a slice of unitary matrix, namely, $\mathbf{H}_{0}^{\dagger} \mathbf{H}_{0}=\mathbf{I}_{r}$, and $\mathbf{R}_{H} \in \mathbb{C}^{r \times r}$ an invertible upper triangular matrix. Then we define a unitary matrix $\mathbf{U}$ that rotates the $r$ orthonormal columns of $\mathbf{H}_{0}$ into the first $r$ elementary vectors, that is,

$$
\mathbf{U H}_{0}=\left[\begin{array}{c}
\mathbf{I}_{r} \\
\mathbf{0}_{(N-r) \times r}
\end{array}\right]
$$

and, in particular,

$$
\mathbf{U M}^{-1 / 2} \mathbf{p}_{0}=\sqrt{\mathbf{p}_{0}^{\dagger} \mathbf{M}^{-1} \mathbf{p}_{0}} \mathbf{e}_{1}
$$

where $\mathbf{e}_{1}$ is the $N$-dimensional column vector whose first entry is equal to one and the remainings are zero. It turns out that the whitened data vector $\mathbf{z}=\mathbf{U M}^{-1 / 2} \mathbf{X}$ is distributed as [28]

$$
\mathbf{z}: \mathcal{C} \mathcal{N}_{N}\left(\alpha \sqrt{\mathbf{p}^{\dagger} \mathbf{M}^{-1} \mathbf{p}}\left[\begin{array}{c}
e^{j \varphi} \cos \phi \\
\mathbf{h}_{B_{0}} \sin \phi \\
\mathbf{h}_{B_{1}} \sin \phi
\end{array}\right], \mathbf{I}_{N}\right),
$$

where $\mathbf{h}_{B_{0}} \in \mathbb{C}^{(r-1) \times 1}, \mathbf{h}_{B_{1}} \in \mathbb{C}^{(N-r) \times 1}$ with

$$
\left\|\left.\mathbf{h}_{B_{0}}\right|^{2}+\right\| \mathbf{h}_{B_{1}} \|^{2}=1
$$

where $\|\cdot\|$ denotes the Euclidean norm of a vector. Then because of the useful signal components, the distributions of $t, b$ and $c$ are given in [23]:

(i) given $b$ and $c, \hat{t}_{\text {glrt }}$ is ruled by the complex noncentral F-distribution with $1, K-N+1$ degrees of freedom and noncentrality parameter

$$
\delta_{\phi}^{2}=\frac{\mathrm{SNR} \cos ^{2} \phi}{1+b+c+b c},
$$

namely, $\hat{t}_{\mathrm{glrt}} \sim \mathcal{C} \mathcal{F}_{1, K-N+1}\left(\delta_{\phi}\right)$;

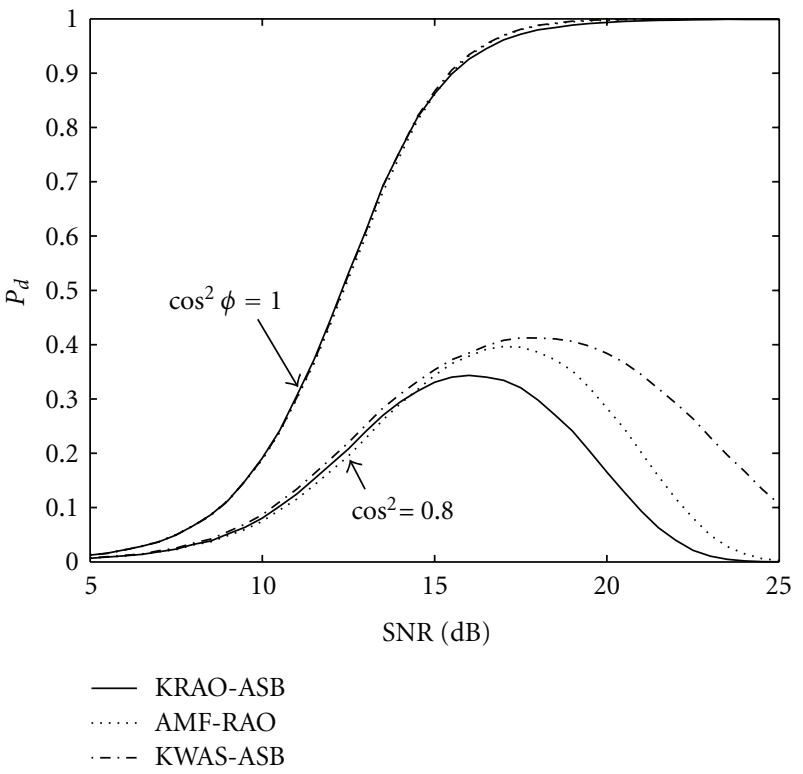

FIgure 8: $P_{d}$ versus SNR for the KRAO-ASB with $\rho=1.2$, the KWAS-ASB with $\gamma=1.1$, and the AMF-RAO, $N=8, K=24$.

(ii) $b$ is a complex noncentral F-distribution rv with $N-$ $r, K-N+r+1$ degrees of freedom and noncentrality parameter

$$
\delta_{b}^{2}=\mathrm{SNR}^{2} \sin ^{2} \phi|| \mathbf{h}_{B_{1}} \|^{2},
$$

namely, $b \sim \mathcal{C} \mathcal{F}_{N-r, K-N+r+1}\left(\delta_{b}\right)$;

(iii) given $b, c$ obeys the complex noncentral Fdistribution with $r-1, K-N+2$ degrees of freedom and noncentrality parameter

$$
\delta_{c}^{2}=\frac{\mathrm{SNR} \sin ^{2} \phi|| \mathbf{h}_{B_{0}} \|^{2}}{1+b},
$$

namely, $c \sim \mathcal{C} \mathcal{F}_{r-1, K-N+2}\left(\delta_{c}\right)$.

Now, it is easy to see that the $P_{d}$ for the SKRAO-ASB can be expressed as

$$
\begin{aligned}
P_{d}(\phi)=P\left[\hat{t}_{s d}>\hat{\eta}_{s}, t_{\text {rao }}>\eta_{r} ; H_{1}\right] \\
=\iint_{0}^{\infty}\left[1-F_{1}\right. \\
\left.\quad \times\left(\max \left(\frac{\hat{\eta}_{s}}{1+\kappa}-1, \frac{\eta_{k}}{(1+\varepsilon+k+\varepsilon k)^{1-2 \rho}-\eta_{k}}\right)\right)\right] \\
\quad \times f_{c \mid b}(\kappa \mid b=\varepsilon) f_{b}(\varepsilon) d \varepsilon d \kappa,
\end{aligned}
$$

where $f_{b}(\cdot)$ is the pdf of the $\mathrm{rv} b \sim \mathcal{C} \mathcal{F}_{N-r, K-N+r+1}\left(\delta_{b}\right)$, $f_{c \mid b}(\cdot \mid \cdot)$ is the pdf of the $\operatorname{rv} c \sim \mathcal{C} \mathcal{F}_{r-1, K-N+2}\left(\delta_{c}\right)$, given $b$, and $F_{1}(\cdot)$ is the cdf of $\hat{t}_{\text {glrt }} \sim \mathcal{C} \mathcal{F}_{1, K-N+1}\left(\delta_{\phi}\right)$, given $b$ and $c$.

In Figures 10 and 11, we plot $P_{d}$ versus $\phi$ (measured in degrees) for the SKRAO-ASB and the KRAO-ASB for $N=8$, 


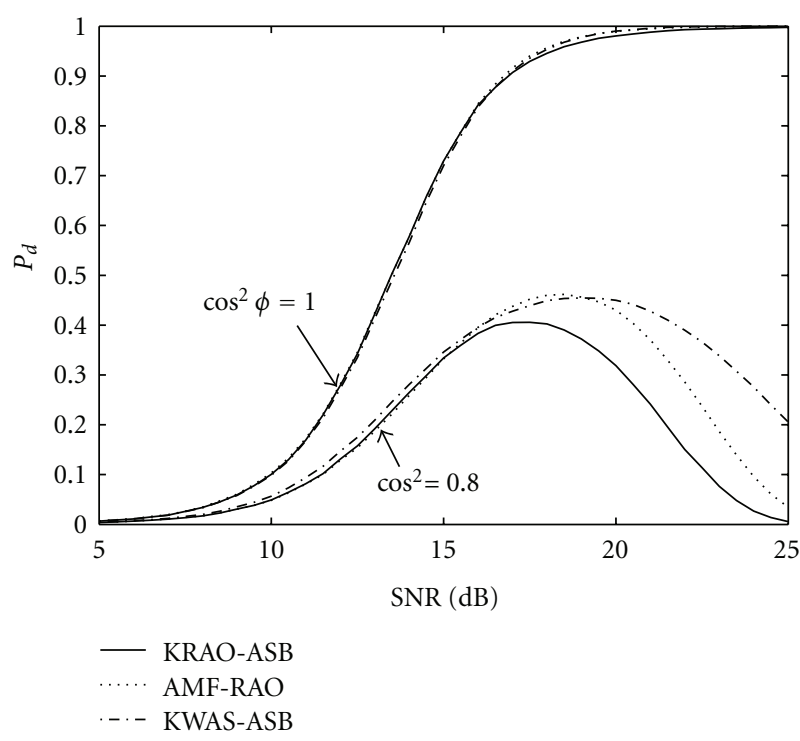

FIgURe 9: $P_{d}$ versus SNR for the KRAO-ASB with $\rho=1.2$, the KWAS-ASB with $\gamma=1.1$, and the AMF-RAO, $N=16, K=32$.

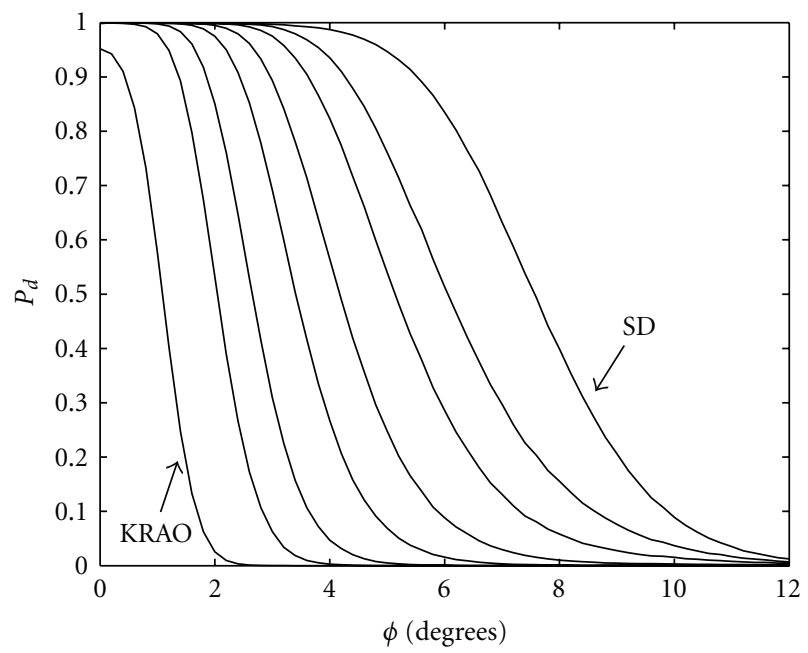

Figure 10: $P_{d}$ versus $\phi$ for the SKRAO-ASB with $N=8, K=24$, $\rho=1.2, \mathbf{H}=[\mathbf{s}(0), \mathbf{s}(\pi / 360)]$, and SNR $=18 \mathrm{~dB}$.

$K=24, \rho=1.2, \mathbf{H}=[\mathbf{s}(0), \mathbf{s}(\pi / 360)], P_{f a}=10^{-4}$, and SNR $=18 \mathrm{~dB}$. The different curves of each plot refer to different threshold pairs. From Figures 10 and 11, it is clear that the SKRAO-ASB can ensure better robustness with respect to the KRAO-ASB, due to the first stage (the $\mathrm{SD}$ ), which is less sensitive than the AMF to mismatched signals. It is also clear that, for a given value of $\rho$, the SKRAO-ASB and the KRAO-ASB exhibit the same capability to reject side lobe signals, due to fact that the second stage (the KRAO) is the same.

Finally, we compare the SKRAO-ASB and the KRAO-ASB in terms of computational complexity. We focus on the first stage of each detector, since the second stage of each detector is to be computed only if the fist stage declares a detection. Observe that the AMF does not require the on-line inversion

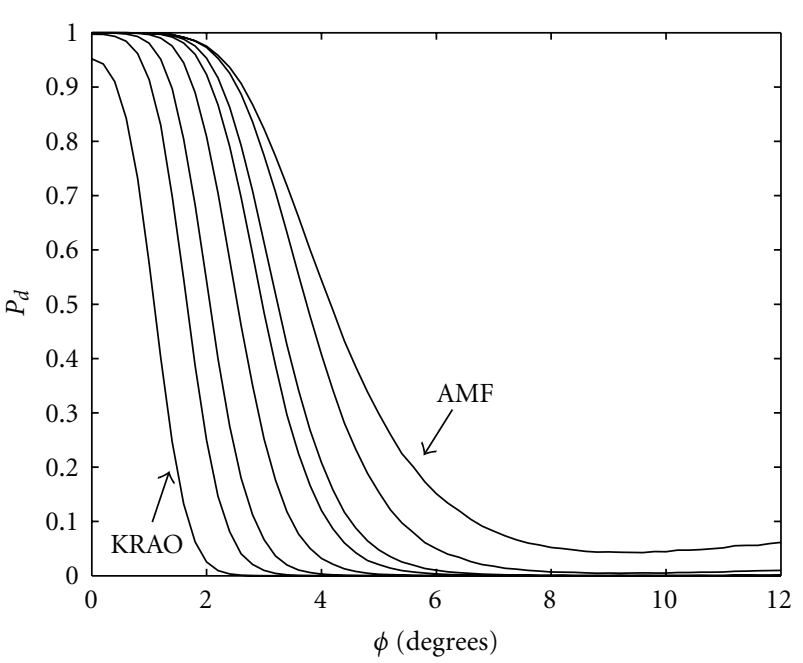

Figure 11: $P_{d}$ versus $\phi$ for the KRAO-ASB with $N=8, K=24$, $\rho=1.2$, and $\mathrm{SNR}=18 \mathrm{~dB}$.

of the matrix $\mathbf{H}^{\dagger} \boldsymbol{S}^{-1} \mathbf{H}(r>1)$ and the computation of the extra term $1+\mathbf{x}^{\dagger} \mathbf{S}^{-1} \mathbf{x}$, which are necessary to implement the SD decision statistic. It is thus apparent that the KRAOASB is faster to implement than the SKRAO-ASB. Anyway, resorting to the usual Landau notation, the SKRAO-ASB involves $O\left(K N^{2}\right)+O(N)$ floating-point operations (flops), whereas the KRAO-ASB requires $O\left(K N^{2}\right)$ flops.

\section{Conclusions}

In this paper, we consider the problem of adaptive signal detection in the presence of Gaussian noise with unknown covariance matrix. Contributions in this paper are summarized as follows.

(i) We propose a new parametric radar detector, KRAO, by merging the statistics of the Kelly's GLRT test and of the Rao test. We discuss its invariance and CFAR property. We derive the closed-form expressions for the probability of false alarm and the probability of detection in matched and mismatched cases.

(ii) We demonstrate performance of KRAO via simulations. Numerical results show that, with a properly selected value for the design parameter, the proposed KRAO can yield better rejection capabilities of mismatched signals than its counterparts. However, when the sensitivity parameter is greater than or equal to unity, it has a nonnegligible loss for matched signals compared with Kelly's GLRT.

(iii) To compensate the matched detection performance of the KRAO, we propose a two-stage detector consisting of an adaptive matched filter followed by the KRAO. We show that such a two-stage detector has desirable property in terms of selectivity. Its invariance and CFAR property have been studied.

(iv) To increase the robustness of the aforementioned two-stage detector, we introduce another two-stage 
detector by cascading a GLRT-based subspace detector and the KRAO. It possesses the CFAR property with respect to the unknown covariance matrix of the noise and it can guarantee a wider range of directivity values with respect to aforementioned two-stage detector.

Further work will involve the analysis of the proposed tunable receivers in a partially homogeneous (Gaussian) environment scenario, that is, when the noise covariance matrices of the primary and the secondary data have the same structure but are at different power levels. It is also needed to investigate these tunable receivers in a clutterdominated non-Gaussian scenario.

\section{Appendix}

\section{Stochastic Representations of the KRAO and the SD}

In this appendix, we come up with suitable stochastic representations for $t_{\text {krao }}$ and $\hat{t}_{s d}$. First, we can recast $t_{\text {krao }}$ as follows:

$$
t_{\mathrm{krao}}=\beta^{2 \rho-1} \frac{\hat{t}_{\mathrm{glrt}}}{1+\hat{t}_{\mathrm{glrt}}},
$$

where $\beta$ is given by (9). It is shown that $\beta$ is distributed as a complex noncentral beta rv [28] and can be expressed as the functions of two independent rv's $b$ and $c$ [21], that is,

$$
\beta=\frac{1}{1+b+c+b c} .
$$

It follows that $t_{\text {krao }}$ can be recast as

$$
t_{\text {krao }}=\left(\frac{1}{1+b+c+b c}\right)^{2 \rho-1} \frac{\hat{t}_{\mathrm{glrt}}}{1+\hat{t}_{\mathrm{glrt}}} .
$$

As to the GLRT-based subspace detector, it is shown that [21]

$$
\hat{t}_{s d}=(1+c)\left(\hat{t}_{\mathrm{glrt}}+1\right) .
$$

A deeper discussion on the statistical characterization of $b$ and $c$ can be found in [23].

\section{Acknowledgments}

The authors are very grateful to the anonymous referees for their many helpful comments and constructive suggestions on improving the exposition of this paper. This work was supported by the National Natural Science Foundation of China under Grant no. 60802072.

\section{References}

[1] E. J. Kelly, "An adaptive detection algorithm," IEEE Transactions on Aerospace and Electronic Systems, vol. 22, no. 2, pp. 115-127, 1986.
[2] F. C. Robey, D. R. Fuhrmann, E. J. Kelly, and R. Nitzberg, "A CFAR adaptive matched filter detector," IEEE Transactions on Aerospace and Electronic Systems, vol. 28, no. 1, pp. 208-216, 1992.

[3] M. Greco, F. Gini, and M. Diani, "Robust CFAR detection of random signals in compound-Gaussian clutter plus thermal noise," IEE Proceedings: Radar, Sonar and Navigation, vol. 148, no. 4, pp. 227-232, 2001.

[4] A. Younsi, M. Greco, F. Gini, and A. M. Zoubir, "Performance of the adaptive generalised matched subspace constant false alarm rate detector in non-Gaussian noise: an experimental analysis," IET Radar, Sonar and Navigation, vol. 3, no. 3, pp. 195-202, 2009.

[5] A. de Maio, G. Alfano, and E. Conte, "Polarization diversity detection in compound-Gaussian clutter," IEEE Transactions on Aerospace and Electronic Systems, vol. 40, no. 1, pp. 114$131,2004$.

[6] X. Shuai, L. Kong, and J. Yang, "Performance analysis of GLRT-based adaptive detector for distributed targets in compound-Gaussian clutter," Signal Processing, vol. 90, no. 1, pp. 16-23, 2010.

[7] E. Conte, A. de Maio, and G. Ricci, "GLRT-based adaptive detection algorithms for range-spread targets," IEEE Transactions on Signal Processing, vol. 49, no. 7, pp. 1336-1348, 2001.

[8] A. de Maio, L. Landi, and A. Farina, "Adaptive radar detection in the presence of mutual coupling and near-field effects," IET Radar, Sonar and Navigation, vol. 2, no. 1, pp. 17-24, 2008.

[9] N. B. Pulsone and C. M. Rader, "Adaptive beamformer orthogonal rejection test," IEEE Transactions on Signal Processing, vol. 49, no. 3, pp. 521-529, 2001.

[10] E. Conte, M. Lops, and G. Ricci, "Asymptotically optimum radar detection in compound-Gaussian clutter," IEEE Transactions on Aerospace and Electronic Systems, vol. 31, no. 2, pp. 617-625, 1995.

[11] S. Kraut and L. L. Scharf, "The CFAR adaptive subspace detector is a scale-invariant GLRT," IEEE Transactions on Signal Processing, vol. 47, no. 9, pp. 2538-2541, 1999.

[12] F. Bandiera, O. Besson, and G. Ricci, "An ABORT-like detector with improved mismatched signals rejection capabilities," IEEE Transactions on Signal Processing, vol. 56, no. 1, pp. 1425, 2008.

[13] F. Bandiera, O. Besson, D. Orlando, and G. Ricci, "Theoretical performance analysis of the W-ABORT detector," IEEE Transactions on Signal Processing, vol. 56, no. 5, pp. 2117-2121, 2008.

[14] M. Greco, F. Gini, and A. Farina, "Radar detection and classification of jamming signals belonging to a cone class," IEEE Transactions on Signal Processing, vol. 56, no. 5, pp. 19841993, 2008.

[15] A. de Maio, "Robust adaptive radar detection in the presence of steering vector mismatches," IEEE Transactions on Aerospace and Electronic Systems, vol. 41, no. 4, pp. 1322-1337, 2005.

[16] O. Besson, "Detection of a signal in linear subspace with bounded mismatch," IEEE Transactions on Aerospace and Electronic Systems, vol. 42, no. 3, pp. 1131-1139, 2006.

[17] F. Bandiera, A. de Maio, and G. Ricci, "Adaptive CFAR radar detection with conic rejection," IEEE Transactions on Signal Processing, vol. 55, no. 6, pp. 2533-2541, 2007.

[18] A. de Maio, "Rao test for adaptive detection in Gaussian interference with unknown covariance matrix," IEEE Transactions on Signal Processing, vol. 55, no. 7, pp. 3577-3584, 2007.

[19] C. D. Richmond, "Performance of a class of adaptive detection algorithms in nonhomogeneous environments," IEEE 
Transactions on Signal Processing, vol. 48, no. 5, pp. 1248-1262, 2000.

[20] C. D. Richmond, "Performance of the adaptive sidelobe blanker detection algorithm in homogeneous environments," IEEE Transactions on Signal Processing, vol. 48, no. 5, pp. 12351247, 2000.

[21] F. Bandiera, D. Orlando, and G. Ricci, "A subspace-based adaptive sidelobe blanker," IEEE Transactions on Signal Processing, vol. 56, no. 9, pp. 4141-4151, 2008.

[22] F. Bandiera, O. Besson, D. Orlando, and G. Ricci, "A two-stage detector with improved acceptance/rejection capabilities," in Proceedings of the IEEE International Conference on Acoustics, Speech and Signal Processing (ICASSP '08), pp. 2301-2304, Las Vegas, Nev, USA, April 2008.

[23] F. Bandiera, O. Besson, D. Orlando, and G. Ricci, "An improved adaptive sidelobe blanker," IEEE Transactions on Signal Processing, vol. 56, no. 9, pp. 4152-4161, 2008.

[24] S. Z. Kalson, "An adaptive array detector with mismatched signal rejection," IEEE Transactions on Aerospace and Electronic Systems, vol. 28, no. 1, pp. 195-207, 1992.

[25] F. Bandiera, D. Orlando, and G. Ricci, "One- and two-stage tunable receivers," IEEE Transactions on Signal Processing, vol. 57, no. 6, pp. 2064-2073, 2009.

[26] S. Bose and A. O. Steinhardt, "Maximal invariant framework for adaptive detection with structured and unstructured covariance matrices," IEEE Transactions on Signal Processing, vol. 43, no. 9, pp. 2164-2175, 1995.

[27] S. Kraut, L. L. Scharf, and L. T. McWhorter, "Adaptive subspace detectors," IEEE Transactions on Signal Processing, vol. 49, no. 1, pp. 1-16, 2001.

[28] E. J. Kelly, "Adaptive detection in non-stationary interference-part III," Tech. Rep. 761, MIT, Lincoln Laboratory, Lexington, Mass, USA, August 1987. 